\title{
Analysis of Early Impact of COVID-19 on Presentation and Management of Oral Cancers - an Experience from a Tertiary Care Hospital in South India
}

\author{
Jeyashanth Riju ${ }^{1}$ (D) Amit Jiwan Tirkey ${ }^{1} \cdot$ Manu Mathew $^{2} \cdot$ Gaurav Chamania $^{1} \cdot$ Malavika Babu $^{3} \cdot$ Shruthi Patil $^{1}$. \\ Ronald Anto ${ }^{1} \cdot$ Mansi Agarwal ${ }^{1} \cdot$ Konduru Vidya $^{1}$
}

Received: 22 October 2020 / Accepted: 24 February 2021 / Published online: 5 March 2021

(C) Indian Association of Surgical Oncology 2021

\begin{abstract}
Oral cancers are the leading cause of cancer-related death in Indian men. Currently steps to contain the transmission and treatment of COVID-19 pandemic have crippled the entire health care system. With hospitals running short of resources, the oncological practice became standstill, especially during the initial phase. This is a retrospective study among patients who presented to our tertiary care hospital in early 3 months of COVID-19 era(ECE) with respect to pre-COVID-19 era(PCE). The study includes patients discussed in multidisciplinary tumor board(MDT)(421 in ECE Vs 31 in PCE) and those who underwent surgery(192 in ECE Vs 26 in PCE). The presentation and outcomes of oral carcinoma were compared between the two eras. There was a significant drop in the number of patients who presented during ECE. Though mean age and gender remained comparable between groups, there was a statistical difference in relation to demographic profile of patient ( $p$ value $<0.001$ ). Among operated during ECE, $80 \%$ had a significantly advanced tumor stage $(p$ value $<0.034)$ and advanced composite stage $(p$ value $<0.049)$. Among patients discussed in MDT during ECE, $38.7 \%$ were deemed inoperable which is double the number when compared with PCE ( $p$ value $<0.009)$. Results of our
\end{abstract}

\section{Research Highlights}

1. Delaying treatment for an early stage cancer (as proposed by guidelines and consensus) can cause overall deleterious effect not only to patient, and it will also over burden health care system.

2. Eighty percent of patients operated during COVID era had an advanced stage oral cancer compared with $60 \%$ in pre-COVID era.

3. Number of patients deemed inoperable in MDT, during COVID era, were $50 \%$ more than pre COVID era.

4. Drop in inpatient, outpatient, and surgery patient numbers may reflect as acute surge in later part of pandemic.

5. Survival of patients developing oral cancers during COVID era may be worser than pre-COVID era.

Jeyashanth Riju

jjriju@yahoo.co.in

Amit Jiwan Tirkey

ajtirkey@gmail.com

Manu Mathew

parackaldrmanu@gmail.com

Gaurav Chamania

gchamania@gmail.com

Malavika Babu

malavikababu@gmail.com

Shruthi Patil

shruthispv@gmail.com
Ronald Anto

ronaldanto90@gmail.com

Mansi Agarwal

mansiady17@gmail.com

Konduru Vidya

vidya5feb@gmail.com

Department of Head and Neck Surgery, Christian Medical College, Vellore, India

2 Department of Radiotherapy, Christian Medical College, Vellore, India

Department of Biostatistics, CMC, Vellore, India 
study showed a higher incidence of advanced stage disease during ECE, with many patient turning inoperable. Thus, the survival of newly diagnosed oral carcinoma patients will be worser. In the management of oral cancer both early stage and advanced stage should have the same priority. Immediate resumption of safe oncology services is mandatory to curtail the current issues.

Keywords COVID-19 $\cdot$ Pandemic $\cdot$ Oral cancer $\cdot$ Head and neck cancer $\cdot$ Patient demography $\cdot$ Delay $\cdot$ Indian scenario

\section{Introduction}

The emergence of the Coronavirus disease 2019 (COVID19), caused by the severe acute respiratory syndrome coronavirus 2 (SARS-CoV-2), had resulted in an unprecedented global public health crisis. With the World Health Organization (WHO) declaring COVID-19 as a public health emergency on March 11, 2020 [1], many countries had taken stringent efforts to contain the spread virus and for the treatment of the COVID-19. The pandemic resulted in a serious impact on the health care system as many of the other diseases which require intervention tend to be disregarded, because of diversion of resources. Among the diseases, which lost to procure appropriate management, cancers stand tall, with serious implications.

Oral cancers are the leading cause of cancer-related death among men in Indian population. The agestandardized rate of death, due to oral cancer, among men living in low and medium human development index (HDI) is 8.7 per 100,000 male population. India accounts for $36 \%$ of the population living under lower HDI. The cause of disease is mainly attributed to the consumption of tobacco products, areca nut, and alcoholism [2,3].

Any delay in treatment of oral cancer will cause progression of disease. It will render the disease incurable or will necessitate a complex resection and reconstruction and multimodal treatment. A study by Jensen et al. has shown that 1-month delay in treatment of head and neck cancers was associated with a $62 \%$ increase in tumor size and $20 \%$ increase in new nodal metastases, and this eventually upstaged tumor stage in $16 \%$ of the study population [4]. Progression of the disease will ultimately lead to a reduced overall survival and disease-free survival [5].

The existing situation created a road to ruin, due to delay in treatment of oral malignancy. This study was intended to analyze the impact, on oral cancers, during the early phase of COVID 19 pandemic (first 3 months) compared with the previous year. This study will act as a guide to the present trend of disease and will unmask what we are going to face immediate post-COVID era. Data will also act as a reference and will prepare us for future management of oral carcinoma patients. Our approach to oral cancer patients in the early COVID-19 era (ECE) is also explained.

\section{Material and Methods}

This retrospective analysis was carried out at a tertiary care hospital in South India, study period being April 2019 to March 2020 (pre-COVID-19 era, PCE) and April 2020 to June 2020 (ECE). The Institutional Ethical Committee clearance was obtained. All patients who presented to out-patient department, primarily concerned with oral cancers and who underwent primary surgical management for oral cancers with a curative intend were included in the study. Patients who had less than 4 weeks of follow-up were excluded.

One hundred and ninety-two patients underwent surgery for oral cancers in PCE, and 26 patients underwent surgery for oral cancers during ECE. During PCE 421 patients were discussed in the multidisciplinary tumor board (MDT), while during ECE it was only 31 patients. Pathological staging was done according to the American Joint Committee on Cancer (AJCC) 8th staging system for cancer of the oral cavity. All tumors were excised with the aim of achieving a $0.5 \mathrm{~cm}$ clear pathological margin. All patients included underwent neck dissection based on clinical judgment. Reconstruction was done as indicated based on patient factors, but free flaps were avoided during ECE. Following surgery, adjuvant radiotherapy (RT) was planned based on advanced clinical stage or advanced pathological stage or based on risk factors.

On histopathological examination, following various tumor-related factors were considered including pathological tumor stage, nodal stage, composite stage, worst pattern of invasion, and primary resection margin. Lymph nodes were analyzed for nodal stage and lymph node ratio (defined as the ratio between number of pathological cervical lymph nodes and the total number of resected cervical lymph nodes) [5].

\section{Statistical Analysis}

Descriptive statistics such as Mean/Median with SD/IQR were presented for all continuous variables and frequency with percentages were expressed for all categorical variables. To compare the continuous variables in Pre-COVID and Early COVID period, independent $t$ test were used based on the normality assumption. Otherwise corresponding nonparametric method known as Man Whitney was used. Similarly, to compare the categorical variables across the 
ECE, chi-square test was used. $p$ value $<0.05$ was considered as statistically significant variables. All statistical analysis was done by using SPSS software Version 21.

\section{Results}

A tabulation of various impact factors evaluated during COVID 19 scenario is given in Tables 1, 2, 3. Out-patients (OP) visits during PCE were 5904 patients which dropped to 549 (drop of 63\%) during ECE. Nasopharyngoscopy use was limited to patients (a drop of $82 \%$ ) with absolute necessity. Patients presenting back to our center with biopsy-proven recurrence dropped to $74 \%$. The number of in-patients (IP) showed a fall of 51.4\% during ECE (Table 1).

The management of oral carcinoma of patients presenting to our center will be decided after discussion in the MDT (Table 2). There was a decrease in the number of patients discussed during ECE which was $70.5 \%$ less compared with PCE (Fig. 1). Among patients discussed in MDT (Fig. 1) during ECE $38.7 \%$ were inoperable due to advanced-stage at presentation, while in PCE only $19 \%$ were inoperable ( $p$ value of 0.009). Only one patient received neoadjuvant chemotherapy (NACT) during ECE. During ECE $89 \%$ of patients with resectable disease discussed in MDT underwent treatment in our hospital compared with $67 \%$ in PCE, probably because of early available date for surgery or due to the limited availability of medical facilities outside.

Among the patients who underwent surgery (Table 3), the mean age of patients operated during PCE was 51.08 \pm 12.18 years (range $22-91$ years) was comparable to patients operated during ECE $51.27 \pm 12.86$ (range 28-75 years). Seventy-four of included population in PCE were men compared with $65.4 \%$ in ECE. The was no statistical difference between the site of oral cancers at presentation. The effect of lockdown with travel restriction refrained patients presenting from other states which was statistically significant $p$ value $<$ 0.001 .

Although, nasal swab for SARS-CoV-2 was made mandatory for all pre-operative patients, only $54 \%$ (14 of 26 patients) underwent testing in ECE. This was due to lack of testing in

Table 1 General impact during pre COVID era Vs early COVID era

\begin{tabular}{lll}
\hline & $\begin{array}{l}\text { Pre-COVID } \\
\text { Era }\end{array}$ & $\begin{array}{l}\text { Early COVID } \\
\text { Era }\end{array}$ \\
\hline Outpatient visits & 5904 & 549 \\
Inpatient & 288 & 35 \\
Oral cancers operated & 192 & 26 \\
Nasopharyngolaryngoscopy & 972 & 42 \\
Oral biopsy proven recurrence presented & 31 & 2 \\
to OPD & & \\
\hline
\end{tabular}

our center in the initial days. However, none of the preoperative patients tested positive for SARS-CoV-2. One patient tested positive after the completion of radiotherapy and recovered uneventfully. Only patient advised NACT during ECE for borderline operable malignancy later succumbed to COVID-19. There was no statistically significant difference in pathological factors like nodal staging, worst pattern of invasion, or node ratio among the two groups. Significant statistical difference was noted with tumor staging, $73 \%$ tumors in ECE presented in advanced $\mathrm{T}$ stage compared with $51 \%$ in PCE ( $p$ value 0.034 ). With respect to composite stage presentation, $81 \%$ operated in ECE presented with advanced stage compared with $61 \%$ in PCE ( $p$ value 0.049 ) (Fig. 2).

There was no compromise on the quality of surgical resection; thus tumor resection margin and node harvest between ECE and PCE data were comparable. In ECE 55.2\% required modified radical neck dissection as opposed to $27.7 \%$ in PCE ( $p$ value 0.002 ). We avoided accessory procedures like tracheostomy and feeding jejunostomy during ECE. Two of the 26 patients underwent salvage surgery during ECE.

In surgical planning, reconstruction were refined to suite ECE. We stopped performing free flaps. Though many patients presented with advanced disease, we could tailor the defect with local flap (50\% in ECE vs $38 \%$ in PCE) for reconstruction. We consider extubation following surgery ideal and all patients could be extubated. All except one patient required postoperative ICU care in ECE.

There was no statistical difference in adjuvant treatment given to the patient either as neoadjuvant chemotherapy or radiotherapy. Chemoradiotherapy provided in ECE was similar to PCE.

\section{Discussion}

The government imposed a nationwide lockdown in India on March 25, 2020, due to the pandemic. Though our state recorded the first case of the COVID-19 on March 7, 2020, the department of family and health welfare has confirmed infected cases over 90,000 with death toll crossing 1200 , as of June 30,2020 , which is on a steady rise. There was stepwise relaxation in lockdown the public transport facilities which were scarce especially in the initial 3 months $[6,7]$.

We are still in a situation where the demands of these essential services cannot be met because of various factors. Lack of access to the health care system leads to a delay in diagnosis and as well as presentation at an advanced stage. Financial constraints, fear of COVID-19 infection in the hospital and other physical constraints of the patient leading to noncompliance in treatment. Hospitals had to divert resources to SARI (severe acute respiratory illness) wards, to protect the majority of the population, so there was a colossal beds shortage both in the intensive care unit and wards. Many hospitals 
Table 2 Impact on multidisciplinary tumor board decisions

\begin{tabular}{llll}
\hline MDT Decision & $\begin{array}{l}\text { Pre-COVID Era } \\
\text { Number of patients }(n=421)(\%)\end{array}$ & $\begin{array}{l}\text { Early COVID Era } \\
\text { Number of patients }(n=31)(\%)\end{array}$ \\
\hline Unresectable - Palliation & $80(19 \%)$ & $12(38.7 \%)$ & $\mathbf{0 . 0 0 9}$ \\
Neoadjuvant Chemotherapy & $22(5.2 \%)$ & $1(3.2 \%)$ & 0.625 \\
Resectable & $319(75.8 \%)$ & $18(58 \%)$ & $\mathbf{0 . 0 2 9}$ \\
\hline
\end{tabular}

Table 3 Factors involved in operated patients between pre COVID era and early COVID era

\begin{tabular}{|c|c|c|c|c|}
\hline \multicolumn{2}{|l|}{ Factors } & \multirow{2}{*}{$\begin{array}{l}\text { Pre-COVID } \\
\text { Number of patients }(n=192)(\%) \\
51.08 \pm 12.18\end{array}$} & \multirow{2}{*}{$\begin{array}{l}\text { COVID } \\
\text { Number of patients }(n=26)(\%) \\
51.27 \pm 12.86\end{array}$} & \multirow{2}{*}{$\begin{array}{l}\text { P- Value } \\
0.941\end{array}$} \\
\hline Age $($ Mean \pm sd $)$ & & & & \\
\hline Gender & $\begin{array}{l}\text { Male } \\
\text { Female }\end{array}$ & $\begin{array}{l}142(74.0) \\
50(26.0)\end{array}$ & $\begin{array}{l}17(65.4) \\
9(34.6)\end{array}$ & 0.356 \\
\hline Location & $\begin{array}{l}\text { Tamil Nadu } \\
\text { South India, other than Tamil Nadu } \\
\text { Others }\end{array}$ & $\begin{array}{l}23(12.0) \\
10(5.2) \\
159(82.8)\end{array}$ & $\begin{array}{l}12(46.2) \\
3(11.5) \\
11(42.3)\end{array}$ & $<0.001$ \\
\hline Tumor Distribution & $\begin{array}{l}\text { Tongue+FOM } \\
\text { GB complex and lip }\end{array}$ & $\begin{array}{l}102(53.1) \\
90(46.9)\end{array}$ & $\begin{array}{l}10(38.5) \\
16(61.5)\end{array}$ & 0.160 \\
\hline Patholgical Tumor stage & $\begin{array}{l}\mathrm{T} 1 / \mathrm{T} 2 \\
\mathrm{~T} 3 / \mathrm{T} 4\end{array}$ & $\begin{array}{l}94(49.0) \\
98(51.0)\end{array}$ & $\begin{array}{l}7(26.9) \\
19(73.1)\end{array}$ & 0.034 \\
\hline Patholgical Nodal Stage & $\begin{array}{l}\text { N0 } \\
\text { N1/N2 } \\
\text { N3 }\end{array}$ & $\begin{array}{l}117(60.9) \\
57(29.7) \\
18(9.4)\end{array}$ & $\begin{array}{l}16(61.5) \\
7(26.9) \\
3(11.5)\end{array}$ & 0.901 \\
\hline Patholgical composite Stage & $\begin{array}{l}\mathrm{I} / \mathrm{II} \\
\mathrm{III} / \mathrm{IV}\end{array}$ & $\begin{array}{l}75(39.1) \\
117(60.9)\end{array}$ & $\begin{array}{l}5(19.2) \\
21(80.8)\end{array}$ & 0.049 \\
\hline WPOI & $\begin{array}{l}\leq 3 \\
>4\end{array}$ & $\begin{array}{l}45(33.6) \\
89(66.4)\end{array}$ & $\begin{array}{l}6(30.0) \\
14(70.0)\end{array}$ & 0.751 \\
\hline \multicolumn{2}{|l|}{ Ipsilateral Neck Dissection } & $185(96.4)$ & $24(92.3)$ & 0.330 \\
\hline \multicolumn{2}{|l|}{ Contralateral Neck dissection } & $31(16.1)$ & $5(19.2)$ & 0.691 \\
\hline \multicolumn{2}{|l|}{ Post surgery ICU care } & $25(13.0)$ & $1(3.8)$ & 0.176 \\
\hline \multicolumn{2}{|l|}{ Elective tracheostomy } & $9(4.7)$ & $0(0)$ & 0.260 \\
\hline \multicolumn{2}{|l|}{ Feeding gastrostomy } & $4(2.1)$ & $0(0)$ & 0.458 \\
\hline \multicolumn{2}{|l|}{ Immediate extrubation } & $167(87)$ & $26(100)$ & 0.051 \\
\hline \multicolumn{2}{|l|}{ Selective Neck Dissection } & $156(81.3)$ & $13(50)$ & $<0.001$ \\
\hline \multicolumn{2}{|c|}{ Modified Radical Neck Dissection } & $60(31.3)$ & $16(61.5)$ & 0.002 \\
\hline Reconstruction & $\begin{array}{l}\text { No reconstruction } \\
\text { Local flap } \\
\text { PMMC } \\
\text { Free flap }\end{array}$ & $\begin{array}{l}44(22.9) \\
73(38.0) \\
60(31.2) \\
20(10.4)\end{array}$ & $\begin{array}{l}4(15.4) \\
13(50) \\
9(34.6) \\
0\end{array}$ & 0.201 \\
\hline Margin & $\begin{array}{l}\text { Clear } \\
\text { Close } \\
\text { Involved }\end{array}$ & $\begin{array}{l}106(55.2) \\
79(41.1) \\
7(3.6)\end{array}$ & $\begin{array}{l}15(57.7) \\
11(42.3) \\
0(0.0)\end{array}$ & 1.00 \\
\hline \multicolumn{2}{|l|}{ Node Harvest(Mean \pm sd) } & $24.8 \pm 10.0$ & $23.1 \pm 9.8$ & 0.601 \\
\hline Node Ratio & $\begin{array}{l}\leq 0.1 \\
>0.1\end{array}$ & $\begin{array}{l}166(87.4) \\
24(12.6)\end{array}$ & $\begin{array}{l}20(76.9) \\
6(23.1)\end{array}$ & 0.220 \\
\hline Radiotherapy(RT) & $\begin{array}{l}\text { No RT } \\
\text { RT } \\
\text { ChemoRT }\end{array}$ & $\begin{array}{l}38(19.8) \\
125(64.1) \\
29(15.1)\end{array}$ & $\begin{array}{l}6(23.1) \\
16(61.5) \\
4(15.4)\end{array}$ & 0.754 \\
\hline \multicolumn{2}{|c|}{ Neoadjuvant Chemotherapy followed by surgery } & 25 & 2 & 0.439 \\
\hline
\end{tabular}




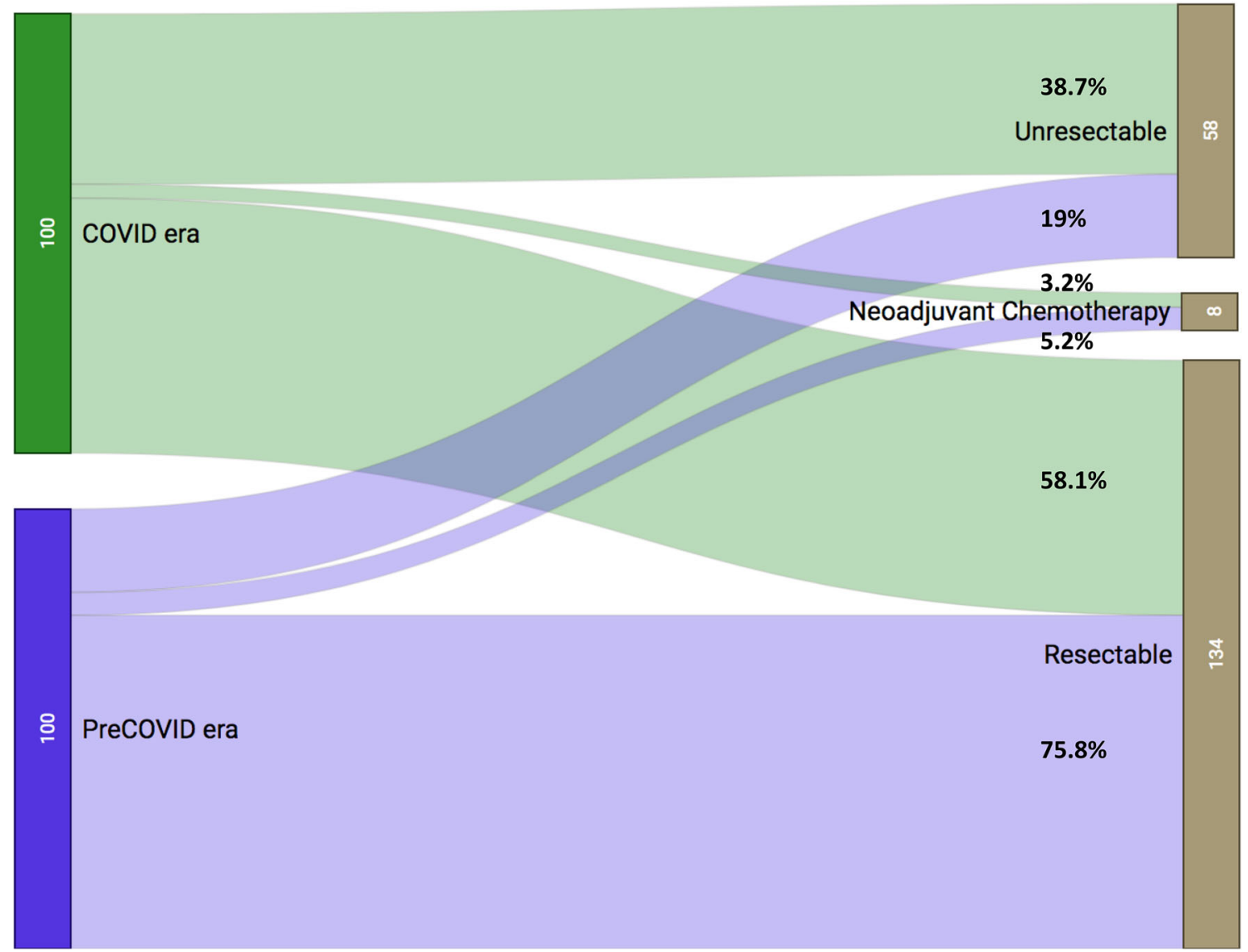

Fig. 1 Sankey diagram demonstrating a high number of inoperable tumors following MDT decisions in early COVID era comparing to pre-COVID era. (Variables expressed as percentage)

had to be converted into COVID-19 care hospitals further limiting oncology practice. Shortage of doctors and nursing staff due to continuous shift in SARI wards, quarantine, and COVID-19 infections not only has cramped the health care system; it further added mental trauma to all health care workers. Another pitfall during early ECE was due to the non-availability of COVID-19 testing facilities, shortage of personal protective equipment, non-availability of negative pressure theater, and shortage of blood products. To overcome challenges there was a tremendous change in approach with regular guidelines proposed in head and neck cancers, which includes American Head and Neck Society (AHNS), Foundation for Head and Neck Oncology, the American College of Surgeons, and the Society of Surgical Oncology $[8,9]$.

The algorithm in Fig. 3 was followed for the maximum benefit of patient, doctors and supporting staff during pandemic. Patients were advised to follow appropriate precaution to prevent COVID-19. For follow-up patients were encouraged to contact us through hospital email, hospital phone numbers and video teleconsultations were started in the late phase. The number of patient visits to our OP during ECE had a steep fall of $63 \%$ and inpatient admission reduced to $51.4 \%$, compared with PCE. We did not stop providing our essential services, for oral cancers, at any point of time during the lockdown. The waiting period for surgery was up to 4 weeks during PCE. Though number of functioning operation theaters decreased with reduction in patients, waiting period was less than 10 days in ECE.

Results shows that there was no change among age and gender among the two groups, but there was a significant difference in patients undergoing treatment based on demographic profiles $(p$ value $<0.001)$. Our hospital not only cater services to the local state population but many from other states of India and also from neighboring countries. Management of all oral cancer patients was directed through MDT. Introduction of teleconferencing came handy, and all members were able to participate through a hospital network. Number of patients turned inoperable after discussing in MDT during ECE and was 50\% more than PCE.

More than $80 \%$ of patients who underwent surgery during ECE had advanced disease and this was 20\% more than PCE. So the tumor resection was also complex, and there was a $30 \%$ rise in the performance of modified radical neck dissection and need for reconstruction raised by $7.6 \%$, during ECE. A prospective cohort study on 27,717 patients with oral cancers showed 5-year overall survival among early-stage oral cancer was around 70-90\% while in those presenting with advanced 


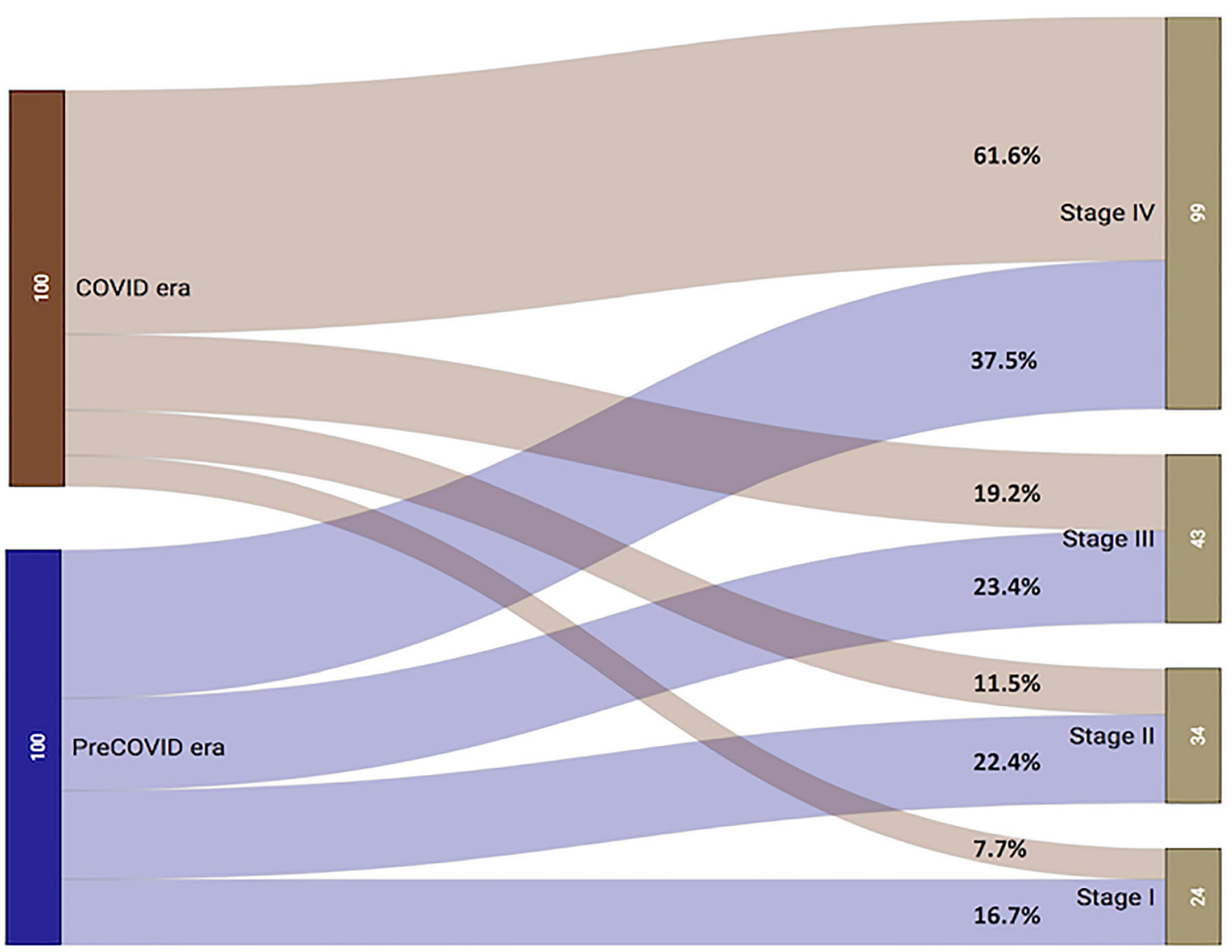

Fig. 2 Sankey diagram demonstrating a advanced stage tumor presentation in early COVID era compared with pre-COVID era. (Variables expressed as percentage in each category)

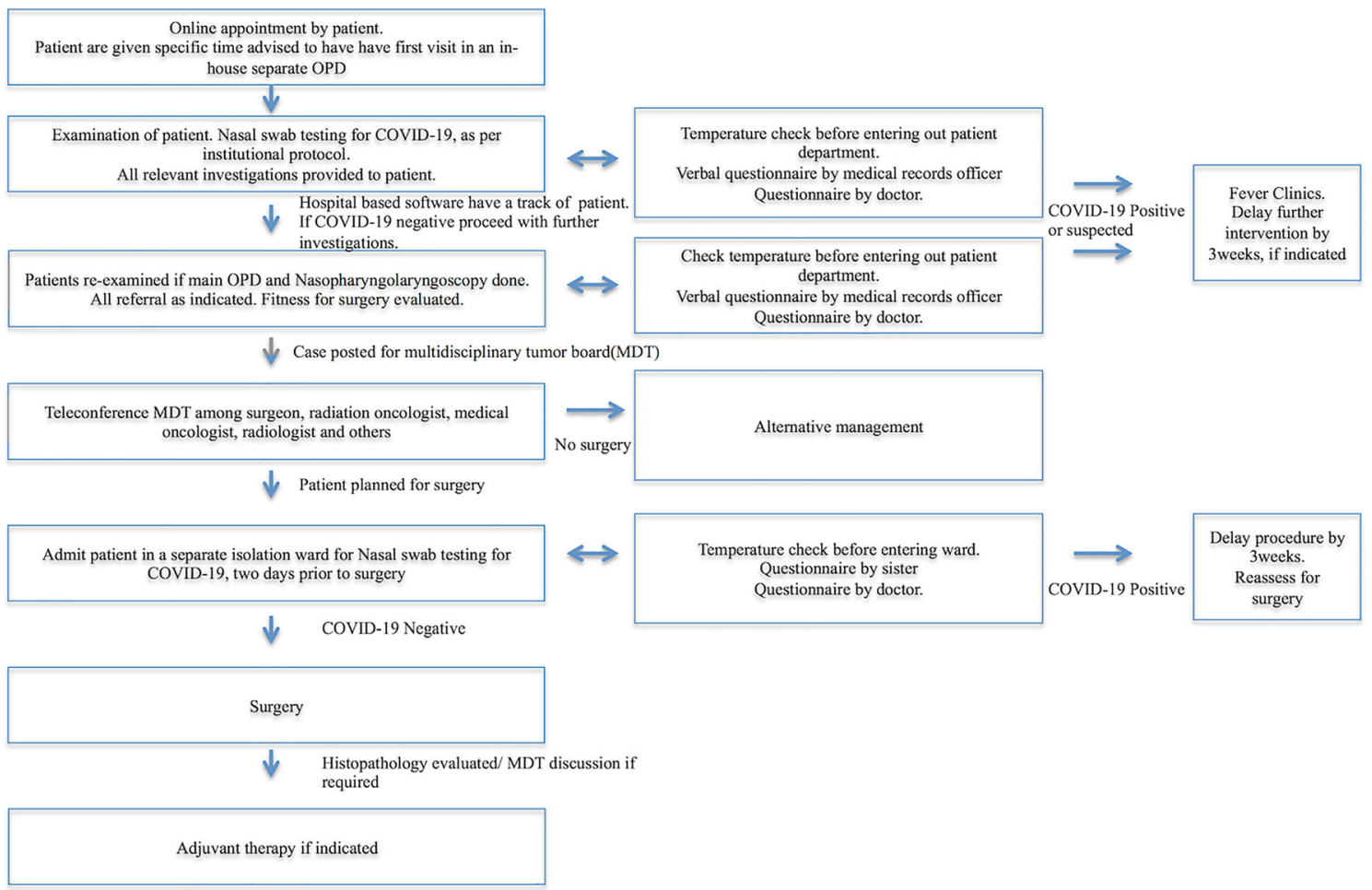

Fig. 3 An effective algorithm followed in management of a new patient with suspected oral cancer during COVID-19 era 
stage was $20-30 \%$ [10]. With more than $80 \%$ patient presenting in advanced stage, there would a clear drop in survival even in operated patients. Our study is based on early 3 months of impact, and the same number could get worser with time. Results clearly point out the direction we are heading with the burden of disease in future. Drop in OP visits in ECE will manifest as an increase in OP visits in later stage as the incidence of oral cancer will remain the same. More importantly, cases are going to present in an advanced stage and the number of cases which cannot be resected will increase. Most hospitals handling oral cancers will face a sudden increase in cases, in future, with prolonged waiting period, especially government sectors.

Early stage oral cancers will require only single-stage procedure like surgery and has a excellent prognosis compared with advanced stage disease which requires a complex surgery and multimodal treatment. So, prioritization of patients with respect to oral cancer, in an Indian scenario, should be taken with caution, as only few cases present early during ECE (19\% as per our study) and the disease progression is fast and with waiting period early disease can easily slip into an advanced disease, which requires aforementioned treatment. There will be a deterioration in quality of life, increased financial burden and prolonged treatment duration for a patient presenting with early oral cancer who has been delayed surgery. Further, the time consumed, budget, and resource allocation for treatment of an advanced staged disease with multimodality might be sufficient to treat more than one early stage disease. Thus early stage and advanced stage diseases should be given the same priority, and if possible early stage cancer should be given top priority.

India has a clear lack in referral centers for cancer treatment especially in the Northern part of India, and the affordability of patients in the private sector remains a question. We could have quickly refrained from the present situation if there were adequate regional cancer centers [11]. But due to lack of sufficient tertiary care cancer hospitals, we could foretell that current cancer centers will be overburdened, and with the same available resources we would struggle to meet the existing demand. Thus prognosis of patients presenting during COVID-19 era is going to be much worse, with poor overall survival and disease-free survival.

International consensus published in June 2020 advised $\mathrm{T} 1 / \mathrm{T} 2$ lesions of the oral cavity to be considered for surgery within 8 weeks of diagnosis, and in advanced head and neck cancers, it is not acceptable to delay surgery beyond 4 weeks of diagnosis. Though the role of NACT is not uniformly supported even in advanced disease, treating any operable disease in a palliative intent was not recommended, with a strong agreement [12]. The surgeries we performed during ECE matched the consensus.

We advised a close follow-up of verrucous lesions of oral cavity with negative biopsy for cancer cells. Oral cancer surgery, be it for a primary tumor or a recurrent tumor, was not delayed, and there was no compromise in the extent of surgery. Neck dissection was done as indicated. The choice of reconstruction was limited only to local flaps and pedicled flaps, though free flap would have been our choice in 3 of patients $(11.5 \%)$ operated during the ECE it was not considered. We avoided free flaps during COVID-19 era mainly because of issues in increased operating time, need for ICU care, and the need for frequent monitoring. We used chisel and hammer for upper alveolectomy, and maxillectomy reciprocating saw was used for other bony works to minimize aerosol generation. The use of drill and burr were contained. Powered instruments like harmonic scalpel or LigaSure ${ }^{\mathrm{TM}}$ was used whenever needed. The use of cautery suction devices can come handy. Negative pressure theater was available for only emergency surgery in COVID-19 patients in our setup. Though most patients presented with advanced disease, we could avoid tracheostomy in all patients. ICU care was limited to one patient for surgery during ECE. There was a limitation for surgical trainees during this period, due to limited surgery and reduced surgical chances.

There was no change in providing adjuvant treatment including chemotherapy, radiotherapy (RT), and chemoradiotherapy. We did not come across a COVID-19 positive patient who required oncological resection, in the initial 3 months, but we had decided if we happened to encounter such a patient then we would delay surgery in those patients by 3 weeks.

Among 192 patients operated during PCE, 38 patients $(19.8 \%)$ preferred RT in hometown but during ECE only one patient of the $26(3.8 \%)$ preferred RT in hometown. Similarly, though $25.2 \%$ (106 of 421 patients) preferred surgery in other centers, following MDT, during PCE only $6.4 \%$ ( 2 of 31 patients) preferred surgery in other centers during ECE. All these further infer results of lockdown and other factors preventing patients to procure treatment in other centers.

The limitation of the study is related to a small number in ECE and retrospective nature. But it clearly appears to quantify as a tell-tale phenomenon of what we face in the current scenario. At current situations survival among newly diagnosed oral cancer patients will be inferior, as a whole during COVID-19 era, due to late stage of presentation, delay in treatment, and further many patient may not be able to present to the hospital because of multiple constraints. Unless we take action mortality due to oral cancer due COVID era will be staggeringly high.

\section{Conclusion}

COVID-19 had resulted in many of unpropitious effects directly and indirectly, with respect to oral cancers. Major failure was a result of lack in access to health care system and 
delay in patient care. This had lead to advanced stage of tumor presentation and patient presenting with an incurable disease. Thus eventually patients who develop oral malignancy during COVID-19 era will have a comparatively short overall survival and disease-free survival. For oral cancer prioritization should be given equal for all stages of disease. Proper prioritization along with planning and immediate resumption of oncology services in a safe way can reduce the current problem. Opening more regional cancer centers as per population needs will definitely help in effective tackling of similar problems in the future.

\section{Declarations}

Financial Disclosure Nil.

Conflict of Interest Nil.

\section{References}

1. Topf MC, Shenson JA, Holsinger FC, Wald SH, Cianfichi LJ, Rosenthal EL, Sunwoo JB (2020) Framework for prioritizing head and neck surgery during the COVID-19 pandemic. Head Neck 42(6):1159-1167

2. Bray F, Ferlay J, Soerjomataram I, Siegel RL, Torre LA, Jemal A (2018) Global cancer statistics 2018: GLOBOCAN estimates of incidence and mortality worldwide for 36 cancers in 185 countries. CA Cancer J Clin 68(6):394-424

3. Peres MA, Macpherson LM, Weyant RJ, Daly B, Venturelli R et al (2019) Oral diseases: a global public health challenge. Lancet. 394(10194):249-260
4. Jensen AR, Nellemann HM, Overgaard J (2007) Tumor progression in waiting time for radiotherapy in head and neck cancer. Radiother Oncol 84(1):5-10

5. Ding D, Stokes W, Eguchi M, Hararah M, Sumner W, Amini A, Goddard J, Somerset H, Bradley C, McDermott J, Raben D, Karam SD (2019) Association between lymph node ratio and recurrence and survival outcomes in patients with oral cavity cancer. JAMA Otolaryngol Head Neck Surg 145(1):53-61

6. Health \& family welfare department government of tamil nadu. COVID-19 Active Cases in Tamil Nadu. [Online]. Available from: https://nhmtn.maps.arcgis.com/apps/opsdashboard/index.html\#/ 095ad0a1c0254b058fa36b32d1ab1977 [Accessed 1 July 2020]

7. Gettleman j, schultz k. Modi Orders 3-Week Total Lockdown for All 13 Billion Indians. [Online]. Available from: https://www. nytimes.com/2020/03/24/world/asia/india-coronavirus-lockdown. html [Accessed 15 June 2020]

8. Gupta A, Arora V, Nair D, Agrawal N, Su YX, et al. (2020) Status and strategies for the management of head and neck cancer during COVID-19 pandemic: Indian scenario. Head Neck 1

9. Patel RJ, Kejner A, McMullen C (2020) Early institutional head and neck oncologic and microvascular surgery practice patterns across the United States during the SARS-CoV-2 (COVID19) pandemic. Head Neck 42(6):1168-1172

10. Su WW, Su CW, Chang DC, Chuang SL, Chen SL et al (2019) Impact of varying anatomic sites on advanced stage and survival of oral cancer: 9-year prospective cohort of 27717 cases. Head Neck 41(5):1475-1483

11. Chandarana MN, Pai PS (2020) Demography and treatment pattern of patients with head and neck carcinoma presenting to a tertiary care center in India: need for urgent decentralization of cancer care. South Asian J Cancer 9(1):38-42

12. Mehanna H, Hardman JC, Shenson JA, Abou-Foul AK, Topf MC, Alfalasi $\mathrm{M}$ et al (2020) Recommendations for head and neck surgical oncology practice in a setting of acute severe resource constraint during the COVID-19 pandemic: an international consensus. Lancet Oncol 21(7):e350-e359

Publisher's Note Springer Nature remains neutral with regard to jurisdictional claims in published maps and institutional affiliations. 